\section{Techniques to Lower CT Dose}

\section{Zoltowski P}

Dose management must be a high priority not only for Coronary Computed Tomography Angiography (CCTA) exams but also for routine studies. As such, it is important that technologists know the available options on the CT scanner to utilize dose reduction techniques.

In fact, due to recent events concerning patient overexposure to CT dose in 2010, the FDA launched The Initiative to Reduce Unnecessary Radiation Exposure from Medical Imaging. Through this initiative, the "FDA is advocating the universal adoption of two principles of radiation protection: appropriate justification for ordering each procedure, and careful optimization of the radiation dose used during each procedure. Each patient should get the right imaging exam, at the right time, with the right radiation dose."1

To ensure the right radiation dose, there are several actions that radiological technologists can employ to offer dosereduction strategies in the imaging suite. These include the following:

\section{Dose reduction strategies}

1. Proper patient positioning on a CT scanner table is the first step to assure use of dose saving options. Higher noise images can occur when patients are not well centered in the scan field of view (FOV). By positioning the body at isocenter, the need to increase $\mathrm{mA}$ to compensate for the noise is eliminated.

2. Utilization of BMI charts: Technologists can reduce dose without image degradation by observing the patient's weight and size. This can be based on the patients' body habitus, or Body Mass Index (BMI). Some radiologists are suggesting that if a patient's BMI is less than 30 , a lower $\mathrm{KVp}$ and $\mathrm{mA}$ can be used.

3. Reducing $\mathrm{mA}$ and $\mathrm{KVp}$ : With $\mathrm{mA}$, the relationship is directly proportional, meaning if $\mathrm{mA}$ is reduced by half, the corresponding dose is reduced by half. If $\mathrm{KVp}$ is reduced, the relationship is nonlinear (exponentially lower). For example, if you reduce $\mathrm{KVp}$ from $120 \mathrm{kVp}$ to 100 $\mathrm{KVp}(16.5 \%$ reduction of $\mathrm{KVp})$ the result is a dose reduction of almost $40 \%$.

4. Limiting the Scan Length/coverage to the ROI: Limiting the coverage of the scan will also help reduce dose. More accurately selecting the coverage area limits the start and end location and, therefore, reduces dose.

5. 3D Dose Modulation (AEC): AEC is an option with the ability to optimize dose to achieve the user inputted image quality (IQ) desired. When set up properly this may provide a dose reduction compared to a fixed $\mathrm{mA}$ scan set up to deliver the same IQ at the highest attenuation locations. 
Before the scan, the technologist selects the desired Noise/IQ. The scanner then automatically tailors the exposure based on the scout and body habitus of the patient.

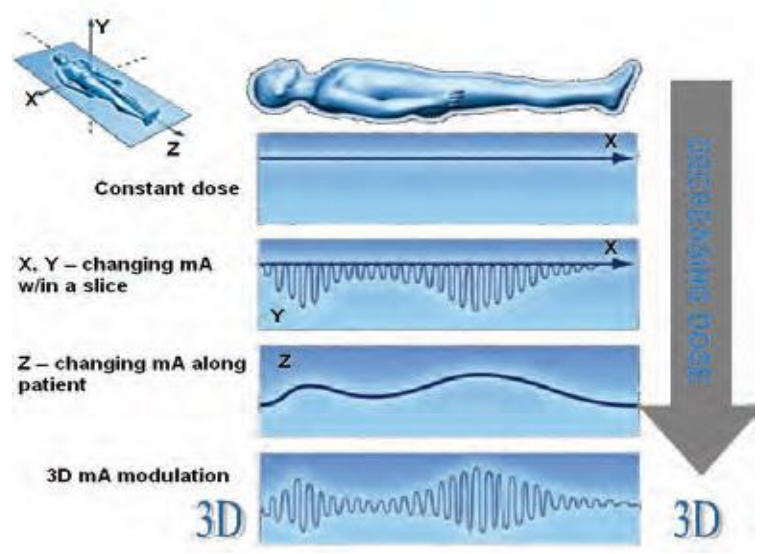

Fig 1: 3D Dose Modulation.

\section{Dose management techniques on GE scanners}

In addition to the actions outlined above, GE provides several dose minimization strategies that are built directly into the scanners. Most of these techniques have been available on GE scanners for many years.

1. AutomA and SmartmA. GE Healthcare's answer for 3D Dose Modulation (AEC) is AutomA* and SmartmA*: AutomA optimizes the $\mathrm{mA}$ for each axial, helical, and cine scan. The benefit is to maintain a constant photon exposure for consistent image quality while providing dose reduction to the patient. AutomA changes $\mathrm{mA}$ across the $\mathrm{z}$ axis while SmartmA changes the $\mathrm{mA}$ across the $\mathrm{x} / \mathrm{y}$ axis within a slice.

2. ECG modulated $\mathrm{mA}$ : When using retrospective gating in the cardiac helical scan mode, radiation dose can be reduced with the use of ECG Modulation. ECG Modulation allows for the variation of $\mathrm{mA}$ across the cardiac cycle depending on the phase of the cardiac cycle where maximum $\mathrm{mA}$ is applied and minimum $\mathrm{mA}$ is modulated across the rest of the phases. The range of phases for peak $\mathrm{mA}$ is prescribed by the user. Depending on the phase range selected for maximum $\mathrm{mA}$, the dose reduction for a cardiac helical scan can be reduced. This technique is not only used on cardiac scans but also on gated PE studies, gated aorta studies and Triple R/O exams to alleviate motion from the heart.

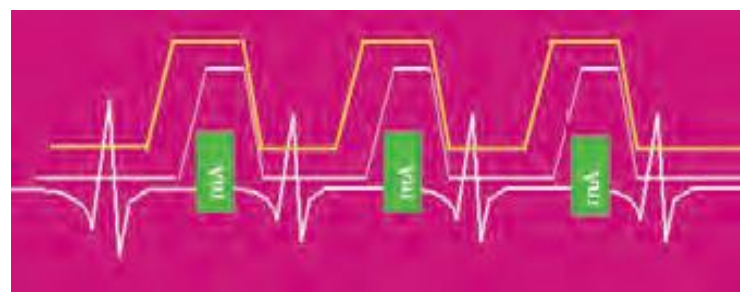

Fig 2: Illustration to show when peak (maximum) $\mathbf{m A}$ is applied.

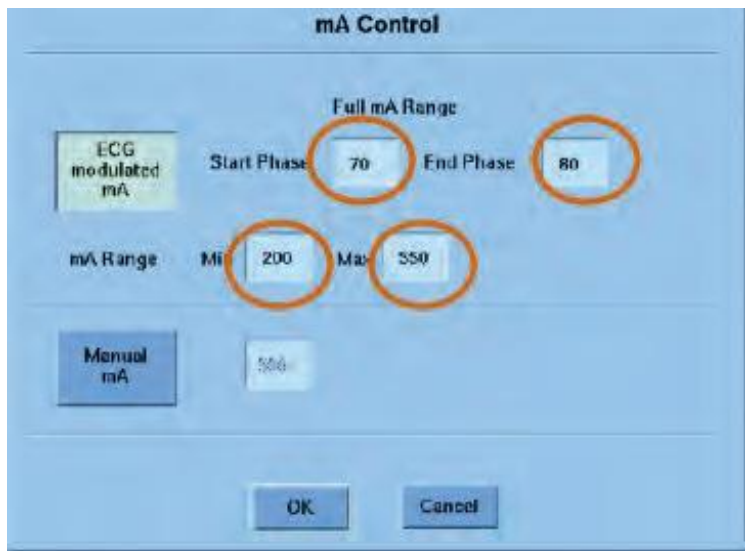

Fig 3: Technologist user interface.

3. Prospective gating: Prospectively gated CCTA utilizing GE Healthcare's SnapShot Pulse dramatically reduces radiation exposure up to $83 \%$ with heart rates below $65 \mathrm{bpm}$ compared to a low pitched helical cardiac acquisition. This is 


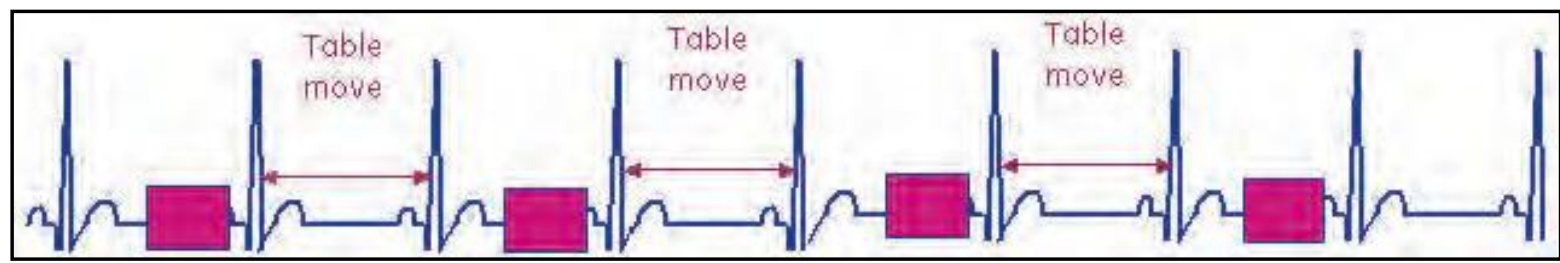

Fig 4: Prospective gating - The pink box displays the specific phase of the cardiac cycle when the $X$-ray is turned on.

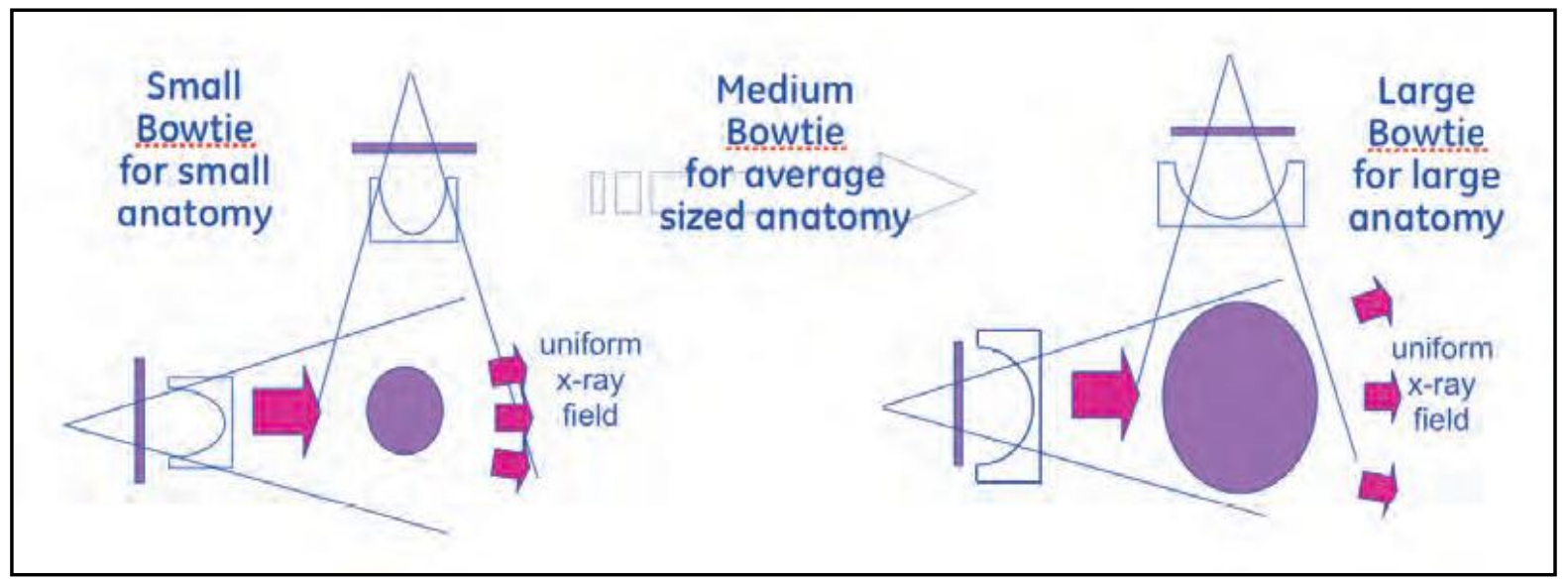

Fig 5: Bowtie beam shaping filter.

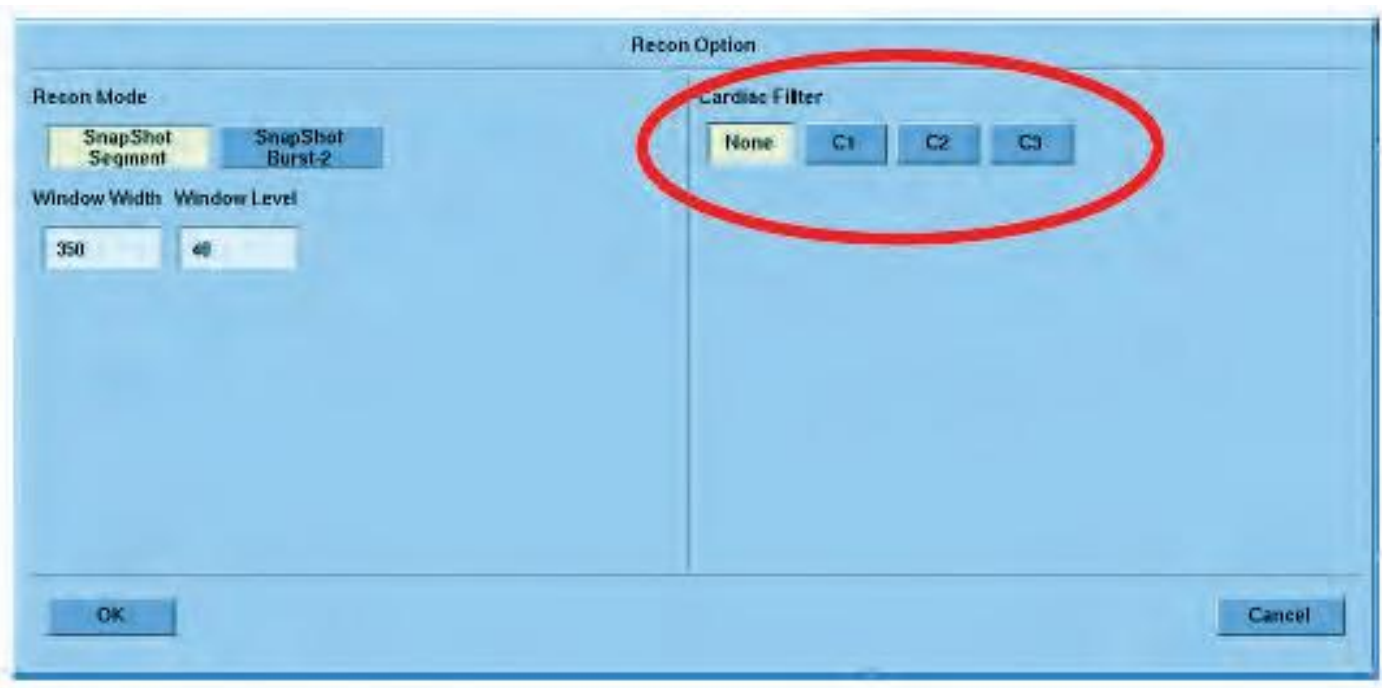

Fig 6: Cardiac filter selection on CT Scanner.

due to the X-ray being on during the prescribed cardiac phases only, meaning that data is not collected continuously across the cardiac cycle. This is an alternative to helical acquisition using axial step-and-shoot mode to acquire images during a specified phase of the heart cycle. This technique captures images of the heart and arteries using a series of three to four exposures taken in a step-and-shoot fashion. The X-ray is turned on only during the prescribed cardiac phases and is turned off when the table moves to the next location.

4. Bowtie Filters: Bowtie beam shaping filters attenuate off-axis rays to minimize dose and reduce X-ray scattering effects. 
GE offers different bowtie filters to more closely match the body part being imaged. Up to three different bowtie filters can be selected to more closely match the body part being images (head, body, heart) and patient size (adult or pediatric). Bowtie filters maintain a more uniform X-ray field at the detector to minimize the surface dose and reduce X-ray scatter. The bowtie filter results in an overall improvement in CT number accuracy, image uniformity, low-contrast detectability and imaging dose.

5. Reconstruction: ASIR (Adaptive Statistical Iterative Reconstruction) is a new reconstruction technique that may enable reduction in image noise (pixel standard deviation). By reducing image noise, ASIR in-turn may allow a technologist to reduce the dose required to produce diagnostic-quality images. ASIR may also enable improvement in low contrast detectability. In clinical practice, the use of ASIR may reduce CT patient dose depending on the clinical task, patient size, anatomical location, and clinical practice. A consultation with a radiologist and a physicist should be made to determine the appropriate dose to obtain diagnostic image quality for the particular clinical task.
6. Cardiac noise reduction filter: Selecting the appropriate cardiac noise reduction filter may also enable the technologist to further reduce dose while preserving the same image quality. This option will not automatically reduce dose. The cardiac noise reducing and edge preserving filters are labeled $\mathrm{C} 1, \mathrm{C} 2$ or $\mathrm{C} 3$. Once the user chooses the appropriate filter, the mA can then be reduced manually.

In addition to utilizing the tools and techniques addressed in this article, it is important for each facility to implement a QA program to review scanning protocols, enact the utilization of pediatric protocols and, most important, as the FDA states in its initiative, ensure the appropriateness of use in CT scanning. To further ensure the safety of patients, facilities should support the continuing education of their technologists, including initial and ongoing certification in CT.

\section{Reference}

1. Available at http:// www.fda.gov/ Radiation- EmittingProducts/ Radiation Safety/RadiationDoseReduction/ucm1999 04.html. 